\title{
Design of Higher Order Delta Segma Modulator for ADC and CMOS Sensors
}

\author{
J. Pushpalatha, G.Neelima
}

\begin{abstract}
This paper presents a 4th-order incremental deltasigma ADC for CMOS image sensors. The ADC employing a cascade of integrators with feed forward (CIFF) architecture uses only one operational transconductance amplifier (OTA) by sharing the OTA between 1st and 2nd stages of the modulator. by using a proposed self-biasing amplifier, which allows active signal summation at the quantizer input node without using an additional OTA, thus power and area savings are achieved. Fabricated in 90nm technology, the 4th orderdsm consumes 32.5 $\mu W$ from a $1.2 \mathrm{~V}$ supply.
\end{abstract}

Keywords : Amplifier sharing, analog-to-digital converter (ADC), cascade of integrators with feedforward (CIFF).

\section{INTRODUCTION}

The regularly expanding interest for high-goals, high edge rate, and low-control CMOS picture sensors (CISs) makes the plan of pixel readout circuits, especially analog to-digital converters (ADCs), a testing undertaking. Albeit single-slant ADCs have been broadly utilized in the section parallel ADC engineering because of their effortlessness, the ADC change time exponentially develops as the ADC goals increments. For instance, a N-bit change requires $2 \mathrm{~N}$ clock cycles, constraining the casing rate of CISs. As of late, delta- sigma $(\Delta \sigma)$ ADCs have effectively shown their handiness for exhibit executions with unrivaled clamor execution and quicker change speed than those of singleincline ADCs. In any case, operational transconductance amplifier (OTAs) utilized in $\Delta \sigma$ modulators regularly take up a lot of intensity and kick the bucket zone. What's more, biasing more than a great many OTAs is certifiably not an inconsequential undertaking. Sharing an inclination voltage through a long metal line between the OTAs makes the predisposition voltage vulnerable to clamor coupling or inadequate settling in the wake of exchanging occasions. Current-mode biasing plans require a large number of metal lines to convey predisposition flows to OTAs and can squander a lot of kick the bucket region and power. In this short, oneself one-sided enhancer proposed in has been adjusted to loosen up the biasing issue. With the end goal to additionally spare power, the amplifier has been altered to be shared by contiguous integrators without being influenced by the remaining charge put away on the intensifier's info parasitic. A second-arrange $\Delta \Sigma A D C$ utilizing the enhancer has been proposed and executed in the $0.18-\mu \mathrm{m}$ CIS process. Despite the fact that it takes up a bite the dust space of just $0.002 \mathrm{~mm} 2$, the ADC accomplishes 10-bit precision and draws the aggregate current of $16.4 \mu \mathrm{A}$

Revised Manuscript Received on September 10, 2019.

J. Pushpalatha, department of ECE, Newtons Institute of Engineering college, Macherla, A.P., India.

G. Neelima, department of ECE, Newtons Institute of Engineering college, Macherla, A.P., India. from a 1.8-V supply, bringing about a figure of merit (FOM) of $151 \mathrm{fJ} /$ change step.

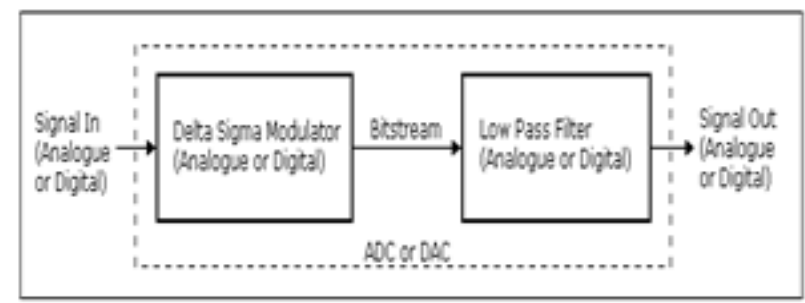

Figure 1- Block Diagram of a Delta Sigma Converter

\section{EXISTING METHOD}

A differential amplifier the yield flag for the most part is the enhanced adaptation of the distinction of two contributions of the intensifier. Due to the selective properties of this sort of intensifier, it is considered as a standout amongst the most essential building obstructs in numerous simple circuits. In this section, we initially investigate a source-coupled circuit as a differential voltageto-current converter and after that bargain with the CMOS differential enhancer in which a current mirror circuit is utilized as a functioning burden for the source-coupled combine. This intensifier is viewed as a basic part at the contribution of most single-finished yield operational speakers with the goal that numerous properties of an operation amp rely upon the parameters of this square. the huge flag attributes of this amplifier in detail. Moreover, the channel length balance impact is disregarded and it is assumed that the deplete current Differential match speaker.

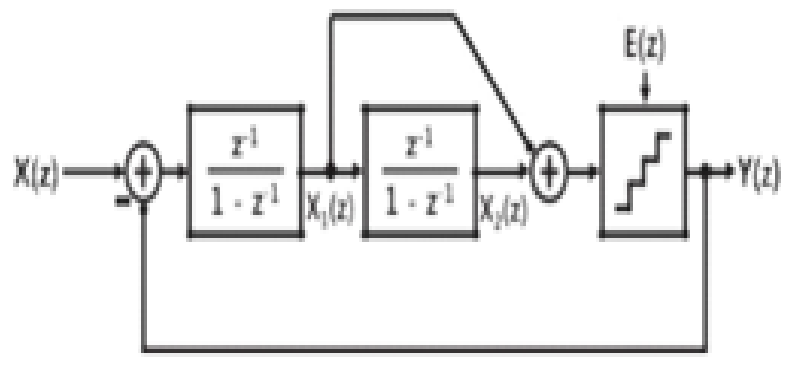

Figure 2: Without an input feedforward path.

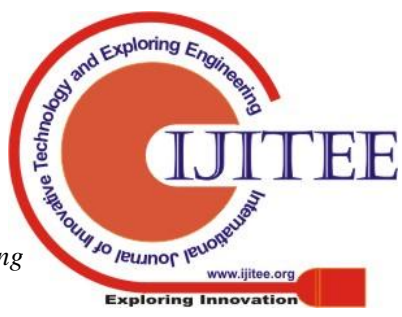




\section{PROPOSED METHOD}

Simple systems have overwhelmed flag preparing for a considerable length of time, yet advanced procedures are gradually infringing into this area. The structure of deltasigma (DS) simple to advanced converters (ADCs) is roughly seventy five percent computerized and one-quarter simple. DS ADCs are currently perfect for changing over simple flags over an extensive variety of frequencies, from DC to a few megahertz. Essentially, these converters comprise of an oversampling modulator pursued by a computerized/demolition channel that together deliver a high-goals information stream yield. This two-section article will take a gander at the DS ADC's center.

A simple flag connected to the contribution of the converter should be moderately moderate so the converter can test it on different occasions, a strategy known as oversampling. The examining rate is multiple times quicker than the computerized outcomes at the yield ports. Every individual example is gathered after some time and - averaged\| with the other info flag tests through the computerized/demolition channel. The DS converter's essential interior cells are the DS modulator and the computerized/pulverization channel. The inward DS modulator coarsely tests the info motion at a high rate into a 1-bit stream. The advanced/pulverization channel at that point takes this tested information and changes over it into a high-goals, slower computerized code. While most converters have one example rate, the DS converter has two - the information sample rate (fS) and the yield information rate (fD). The DS modulator is the core of the DS ADC. It is in charge of digitizing the simple info flag and diminishing clamor at lower frequencies. In this stage, the engineering executes a capacity called commotion forming that pushes low frequency clamor up to higher frequencies where it is outside the band of intrigue. Clamor molding is one reason that DS converters are well suited for low-frequency, high precision estimations. The information flag to the DS modulator is a period differing simple voltage. With the prior DS ADCs, this information voltage flag was essentially for sound applications where AC signals were imperative. Since consideration has swung to accuracy applications, change rates incorporate DC signals. This dialog will utilize a solitary cycle of a sine wave for delineation. Information Acquisition By Bonnie Baker Signal Integrity Engineer $\Delta \sigma$ Modulator Analog Input Digital Filter Decimator Digital Output Digital/Decimation Filter Sample Rate (f ) S Data Rate (fD) f S D/= Decimation Ratio single cycle of a sine wave for the contribution of a DS modulator.

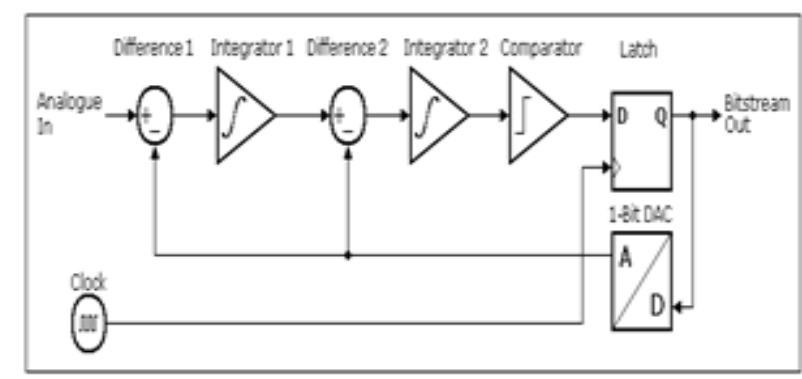

Figure 3.Circuit implementation of the proposed 4th order $\Delta \Sigma$ modulator

As appeared in Fig. 3, the speaker has two inverters associated in parallel whose current is controlled by an upper PMOS (MP) transistor and a lower NMOS (MN) transistor through negative criticism. While one inverter (INV2) enhances an information flag, the other inverter (INV1) builds up the enhancer's predisposition condition. In this brief, as in Fig. 3, two switches, which are driven by two-stage nonoverlapping tickers, are added to enable the inverters to trade jobs. While INV1 fills in as an enhancer for the primary integrator, INV2 sets the predisposition current (Ф2). In the following stage (Ф1), INV2 incorporates the charge from the principal integrator and INV1 sets the inclination current. A schematic of the modulator is appeared in Fig. 4. It is executed in a solitary finished setup, as pixels produce a solitary finished flag. The upper and lower sides of the circuit are the first and second integrators of the modulator, separately. Notice that sharing a speaker between neighboring stages frequently corrupts the linearity of the ADC because of the lingering charge put away on the info parasitic of the intensifier [8]. In any case, this brief does not experience the ill effects of the impact of lingering charge. At the point when INV1 is utilized to set the predisposition current, its information is shorted to a typical mode voltage for information testing.

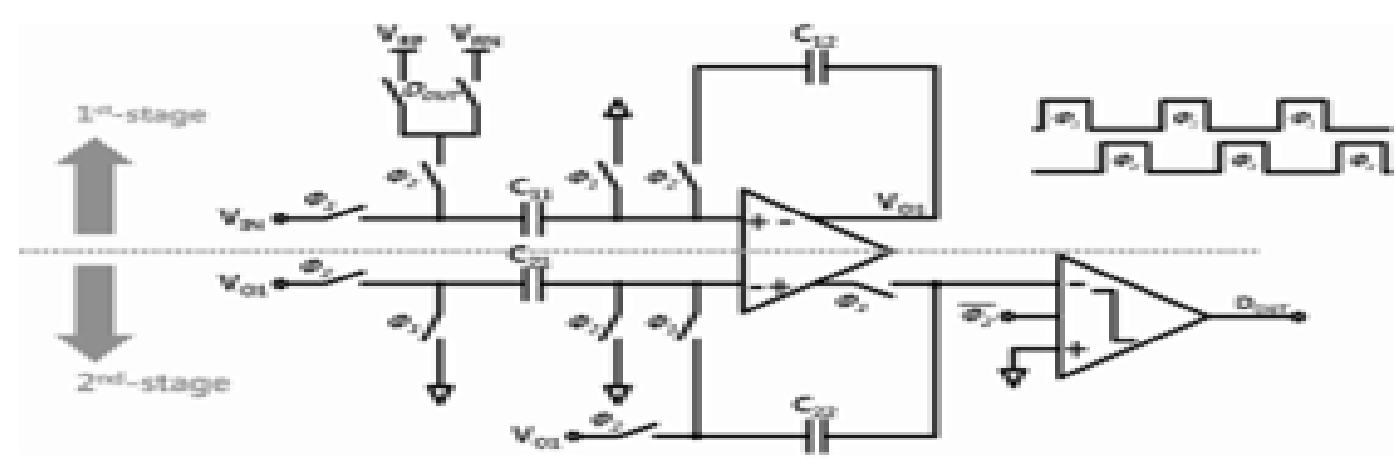

Fig. 4. Circuit implementation of the proposed $\Delta \Sigma$ modulator 

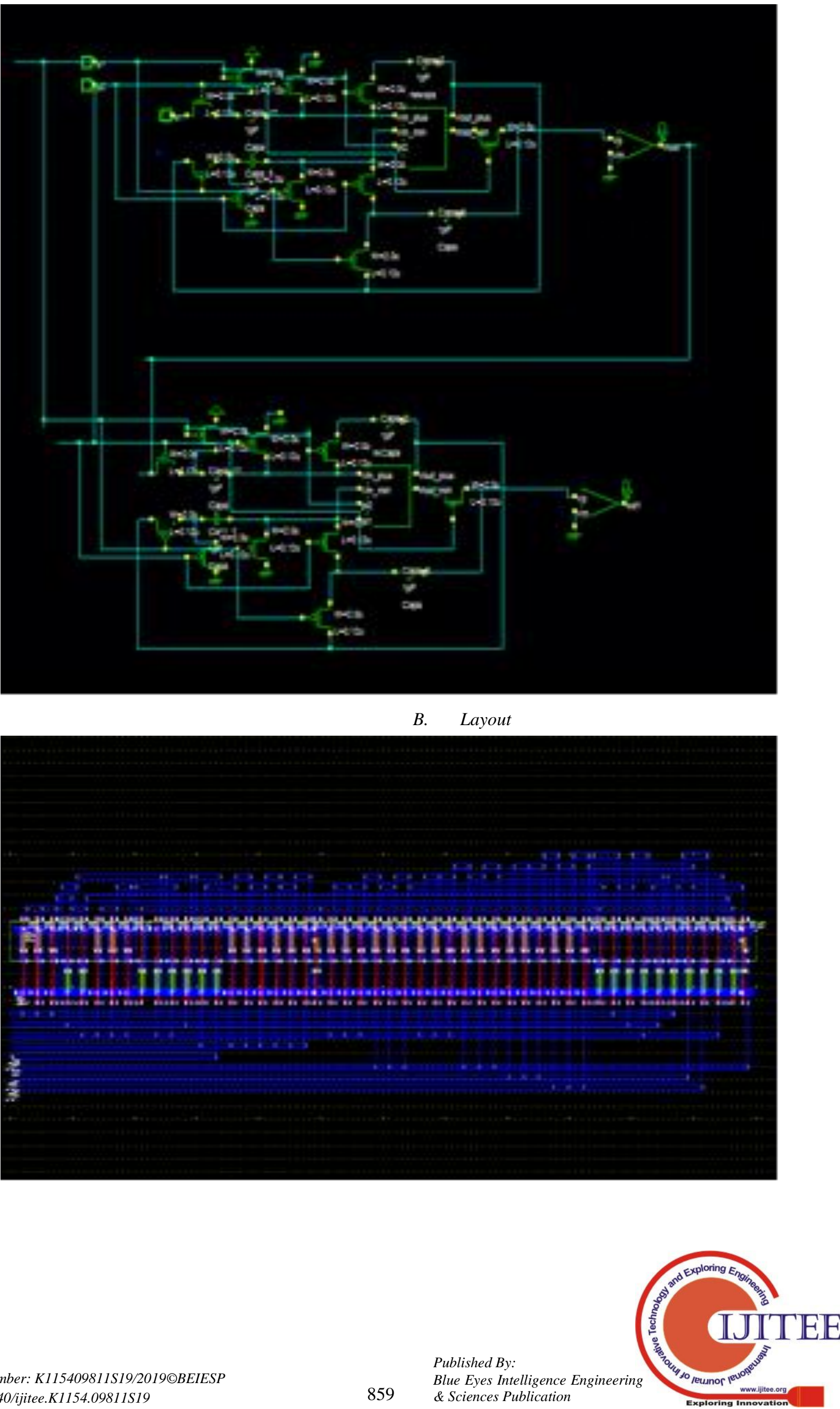
C. Waveforms

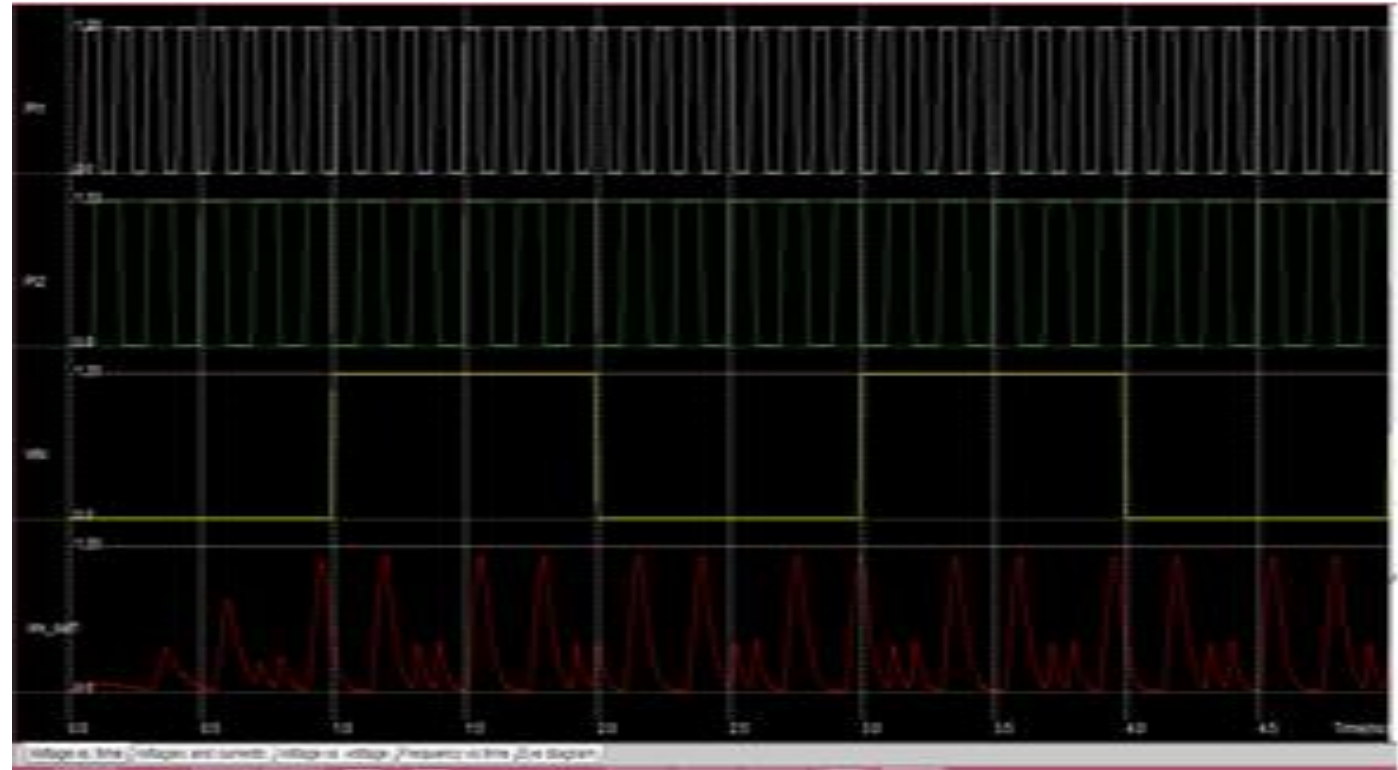

D.

Power Spectral Dencity

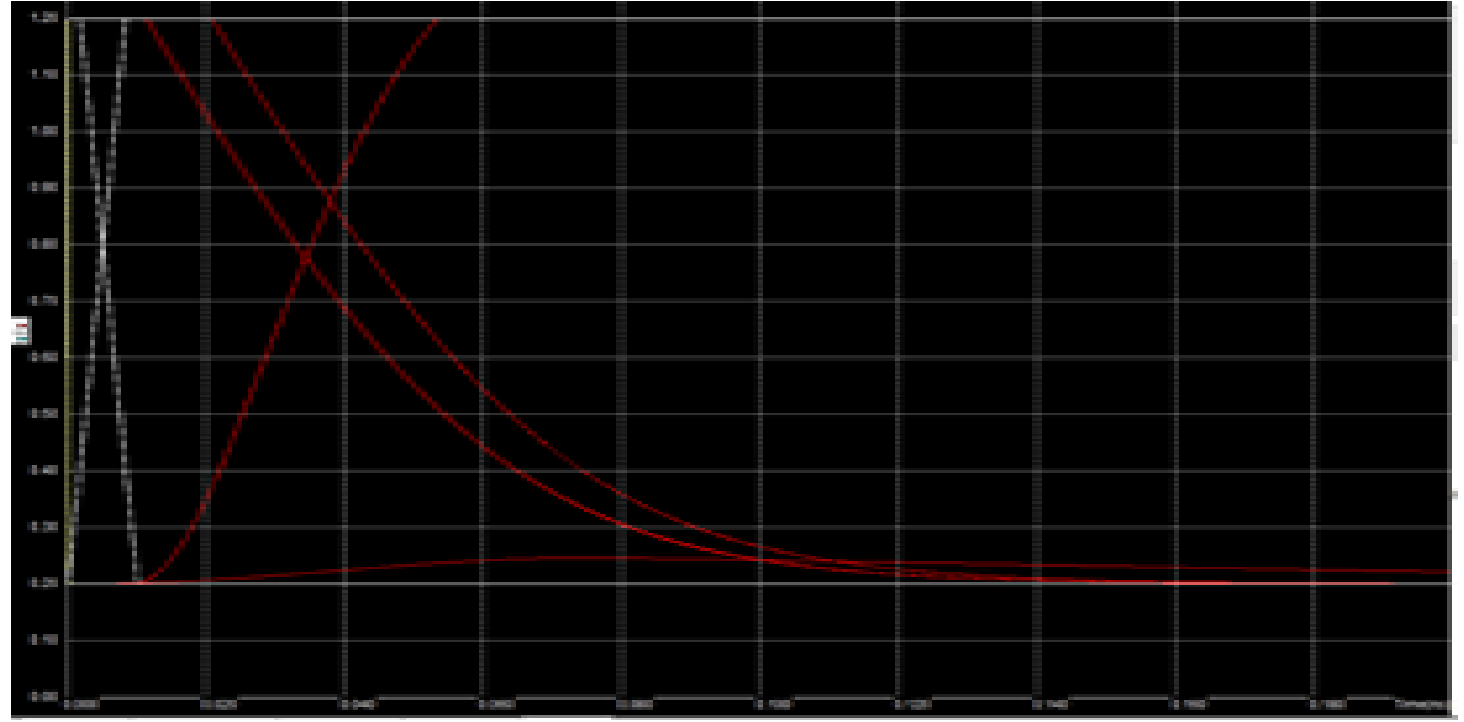

E. V-I characteristics
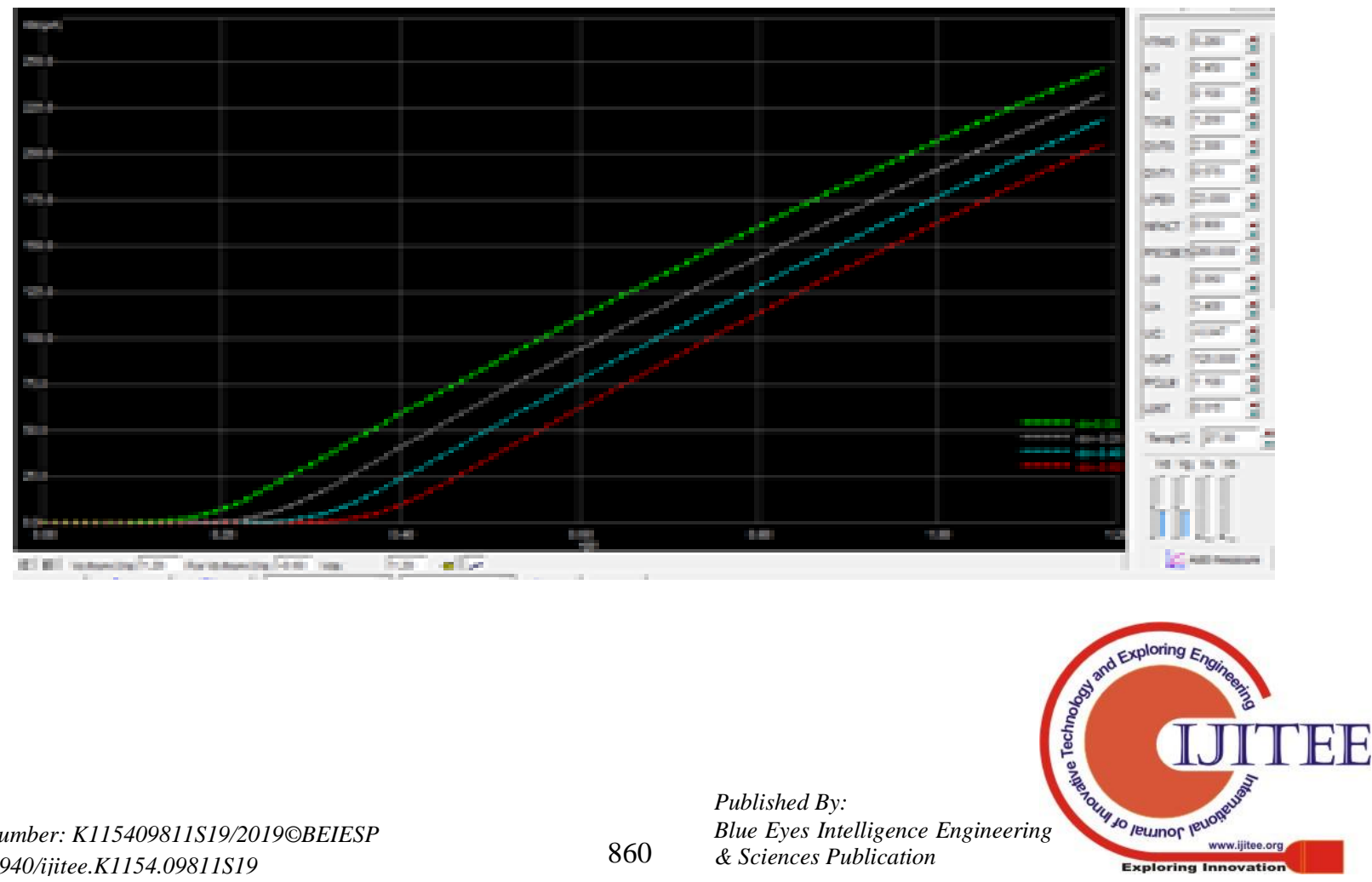


\section{CONCLUSION}

A low-control, little size 10-bit incremental $\Delta \sigma$ ADC for CISs has been proposed and created in the 45-nm CIS innovation. The ADC spares power and kick the bucket territory by sharing a self-biasing intensifier and by utilizing the proposed dimension moving method to perform flag summation..

\section{REFERENCES}

1. S. Yoshihara, Y. Nittaitta, M. Kikuchi, K. Koseki, Y. Ito, Y. Inada, S. Kuramochi, H. Wakabayashi, M. Okano, H. Kuriyama, J. Inutsuka, A. Tajima, T. Nakajima, Y. Kudoh, F. Koga, Y. Kasagi, S. Watanabe, and T. Nomoto, "A 1/1.8-inch 6.4 MPixel 60 outlines/s CMOS Image Sensor With Seamless Mode Change," IEEE J. Strong State Circuits, vol. 41, no. 12, pp. 2998-3006, Dec. 2006

2. M. S. Shin, J. B. Kim, Y. R. Jo, M. K. Kim, B. C. Kwak, H. C. Seol and O. K. Kwon, "CMOS X-Ray Detector With Column-Parallel 14.3-piece Extended-Counting ADCs", IEEE Trans. Electron Dev., vol. 60, no. 3, pp. 1169-1177, Mar. 2013

3. M. Bazes, "Two Novel Fully Complementary SelfBiased CMOS Differential Amplifiers", IEEE J. Strong State Circuits, vol. 26, no. 2, pp. 165-168, Feb. 1991

4. K. Lee, M. R. Mill operator, and G. C. Temes, "A 8.1 $\mathrm{mW}, 82 \mathrm{~dB}$ Delta-Sigma ADC With 1.9 MHz BW and 98 dB THD", IEEE J. Strong State Circuits, vol. 44, no. 8, pp. 2202-2211, Aug. 2009

5. R. Schreier and G. C. Temes, Understanding DeltaSigma Data Con-verters. Piscataway, NJ: Wiley-IEEE Press, 2005. 